Supporting Information for

\title{
Synergistic effect of binary electrolyte on enhancement of the energy density in $\mathrm{Li}-\mathrm{O}_{2}$ batteries
}

Yoko Hase, ${ }^{*} \dagger$ Kiho Nishioka, ${ }^{\ddagger}$ Yasuhiro Komori, $₫$ Takayoshi Kusumoto, ${ }^{\ddagger}$ Juntaro Seki ${ }^{\dagger}$ Kazuhide Kamiya,,$\&$ and Shuji Nakanishi*,\$,\&

${ }^{\dagger}$ Toyota Central R\&D Labs., Inc., 41-1 Yokomichi, Nagakute, Aichi, 480-1192, Japan

* Department of Chemistry, Graduate School of Engineering Science, Osaka University, 1-3 Machikaneyama, Toyonaka, Osaka 560-8531, Japan

$\S$ Research Center for Solar Energy Chemistry, Osaka University, 1-3 Machikaneyama, Toyonaka, Osaka, 560-8531, Japan

*Address correspondence to: y-hase@mosk.tytlabs.co.jp (Y.H); nakanishi@chem.es.osaka-u.ac.jp (S.N)

\section{Materials and Experimental Methods}

Materials. All materials were used as purchased without further purification and stored under argon (Ar). Lithium foil (0.4 mm of thickness) and Carbon paper (CP) (TGP-H-060, $190 \mu \mathrm{m}$ of thickness) were obtained from Honjo Metal and Toray Industries, Inc., respectively. After drying CP cathodes in vacuo at $150{ }^{\circ} \mathrm{C}$ for $5 \mathrm{~h}$, the cathodes were transferred to a glove box without exposure of ambient air. Anhydrous acetonitrile $(\mathrm{MeCN})$ (water content $<0.001 \%$ ) and anhydrous dimethyl sulfoxide (DMSO) (water content $<0.001 \%$ ) were obtained from Wako (Osaka, Japan). Lithium bis(trifluoromethanesulfonyl)imide (LiTFSI) $(99.8 \%$, water content $<0.01 \%)$ was purchased from Tokyo Kasei. Conditioned electrolyte containing 1 M LiTFSI in the mixed solvent of ethylene carbonate and diethyl carbonate (EC and DEC, 3/7, v/v, lithium battery grade) was obtained from Kishida Chemical Co., Ltd. The water content of the prepared electrolytes, measured by Kerl Fischer titration, are $<30 \mathrm{ppm}$.

Electrochemical Measurements. Assembly of cells and introduction of oxygen gas were carried out in the glovebox filled with dry Ar gas, using fully dried instruments and materials. A typical experimental 
procedure is described below. The anode and cathode compartments of the Swagelok-type cell were separated by a solid-state Li ion-conductive glass-ceramic film (LIC-GC, Ohara Inc.) to prevent side reactions between the Li metal and electrolytes applied to the cathode side. The detailed construction of the cell was described in the previous report. ${ }^{1}$ This cell system allows us to employ $\mathrm{MeCN}$ as an electrolyte solvent to a $\mathrm{Li}_{2} \mathrm{O}_{2}$ battery with $\mathrm{Li}$ metal anode, even though $\mathrm{MeCN}$ shows crucially poor reductive stability. $\mathrm{CP}$ was used in a diameter of $20 \mathrm{~mm}$ for a cathode electrode. The average mass of $\mathrm{CP}$ was $0.0260 \mathrm{~g}$ and the specific surface areas were $0.31 \pm 0.20 \mathrm{~m}^{2}{ }_{\mathrm{BET}} / \mathrm{g}$, which were measured by $\mathrm{Kr}$ (99.999\%) physisorption with a BELSOAP-MAX (MicrotracBEL) at $77 \mathrm{~K}$ employing the Brunauer-Emmett-Teller isotherm. $200 \mu \mathrm{L}$ of the binary electrolytes was added into the cathode-side chamber of the cell and high-purity $\mathrm{O}_{2}$ gas $(0.15 \mathrm{MPa},>99.99 \%)$ was introduced into a $50 \mathrm{~mL}$ cylinder which was attached to the cell during the electrochemical measurements. Li metal and 1 M LiTFSI in the mixed solvent of EC and DEC were used as an anode and an electrolyte for the anode-side, respectively. After that, the assembled cells were taken out from the glovebox, and the battery characteristics were determined using an Aska Electronic ACD-01 battery performance analyzer under atmospheric condition at $25{ }^{\circ} \mathrm{C}$. The cell was allowed to rest at open circuit voltage for at least 12 hours before measurements. The galvanostatic discharging experiments were performed to a reductive potential of $1.8 \mathrm{~V}$ at a current density of $0.2 \mathrm{~mA} / \mathrm{cm}^{2}$.

Potentiostatic discharging and linear sweep voltammogram measurement were performed using $\mathrm{Li}-\mathrm{O}_{2}$ cell (2-electrode setup) which was assembled in the same way described above. The potentiostatic discharge characteristics were determined using a Solartron analytical 1400 CellTest System at $25{ }^{\circ} \mathrm{C}$. After the cell was allowed to rest at open circuit voltage for at least 12 hours, the potentials of the cells were immediately shifted to various potentials for the potentiostatic discharging test and potentiostatic discharging was perfomed until the current density reached $0.05 \mathrm{~mA} / \mathrm{cm}^{2}$. Linear sweep voltammogram measurements was performed using Bio-Logic VMP3 system at $25^{\circ} \mathrm{C}$.

Characterization of the Discharged Cathodes. For the characterization of discharged cathodes, the cells were disassembled in an Ar glovebox and the discharged cathodes were extracted from the cells. The cathodes were rinsed with $\mathrm{MeCN}$ for 5 times to remove electrolytes and dried in vacuo at room temperature for $1 \mathrm{~h}$. Scanning Electron Microscopy (SEM) images were obtained using SEM system 
(Hitachi high-technologies, S-4300) at an acceleration voltage of $1 \mathrm{kV}$. The samples were transferred from a glove box to the SEM chamber using a closed vessel to avoid exposure to the air. Powder X-ray diffraction (PXRD) patterns of the discharged cathodes were measured with a powder X-ray diffractometer (Rigaku, UltimaIV) in an air-sensitive sample holder. Measurements were performed at room temperature from 22.5 to $37.5^{\circ} 2 \theta$. The width of sampling and scan speed were $0.1^{\circ}$ and $0.5^{\circ} / \mathrm{min}$., respectively. We did not find any noticeable changes in the PXRD patterns over the measurement time.

Raman scattering spectroscopy. The samples of electrolyte with various composition of $\mathrm{MeCN}$ and DMSO were prepared in the glovebox. The Raman spectra were measured in an airtight quartz cell using a Raman spectrometer (RS-3200, JASCO) with $532 \mathrm{~nm}$ laser excitation, which was calibrated with a diamond standard. The deconvolution analyses were performed using a Gaussian-Lorentzian function on the JASCO spectra manager program after baseline correction.

Pretreatment of cathode electrode for the galvanostatic discharging test of Figure 4d. All pretreatment process was performed in the glovebox as described below. The cathode electrodes of CP (in a diameter of $20 \mathrm{~mm}$ ) were immersed in $\mathrm{MeCN}$ for $1 \mathrm{~min}$ and dried under Ar atmosphere at room temperature and pressure for $10 \mathrm{~min}$. The change of weight before and after preparation was below 0.3 mg per an electrode. The number of monolayers was estimated from the weight of adsorbed $\mathrm{MeCN}$, the calculated volume of a single $\mathrm{MeCN}$ molecule, and the specific surface area of cathode. The pretreated CPs were set in the cathode side chamber of the cell.

\section{References}

(1) Hase, Y.; Komori, Y.; Kusumoto, T.; Harada, T.; Seki, J.; Shiga, T.; Kamiya, K.; Nakanishi, S., Negative differential resistance as a critical indicator for the discharge capacity of lithium-oxygen batteries. Nat. Commun. 2019, 10, 596.

(2) Tatara, R.; Kwabi, D. G.; Batcho, T. P.; Tulodziecki, M.; Watanabe, K.; Kwon, H.-M.; Thomas, M. L.; Ueno, K.; Thompson, C. V.; Dokko, K.; Shao-Horn, Y.; Watanabe, M., Oxygen Reduction Reaction in Highly Concentrated Electrolyte Solutions of Lithium Bis(trifluoromethanesulfonyl)amide/Dimethyl Sulfoxide. J. Phys. Chem. C 2017, 121, 9162-9172. 
(a)

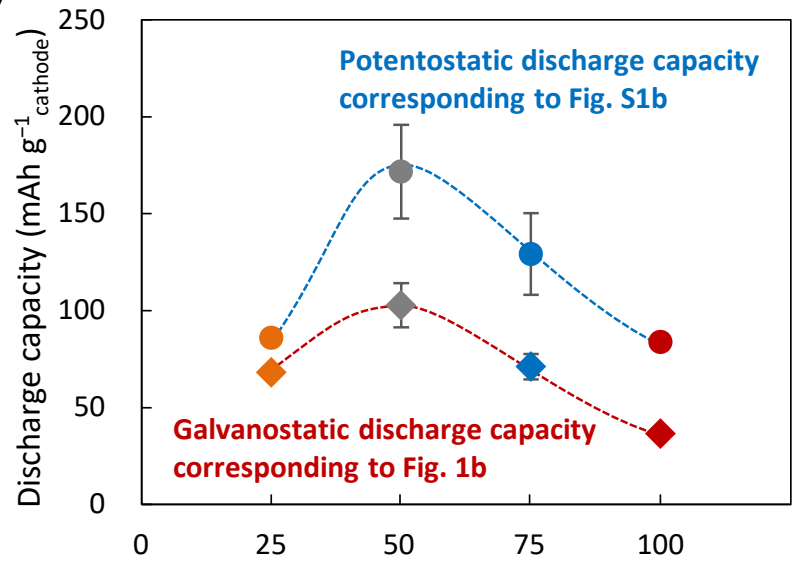

Content of DMSO in the electrolyte solvents (vol\%)

(b)

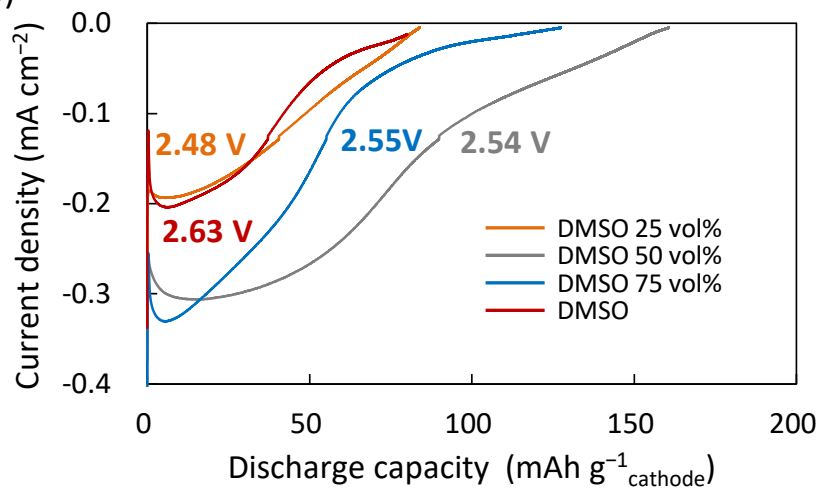

Figure S1. (a) Discharge capacities corresponding to Figures $1 \mathrm{~b}$ (diamonds) and S1b (circles).(b) Potentiostatic discharging curves obtained in the binary solvent electrolytes including $0.5 \mathrm{M}$ LiTFSI. The applied cell voltages were corresponding to the cell voltages at $0.2 \mathrm{~mA} \mathrm{~cm}^{-2}$ in the LSV measurement (Figure 1b). 


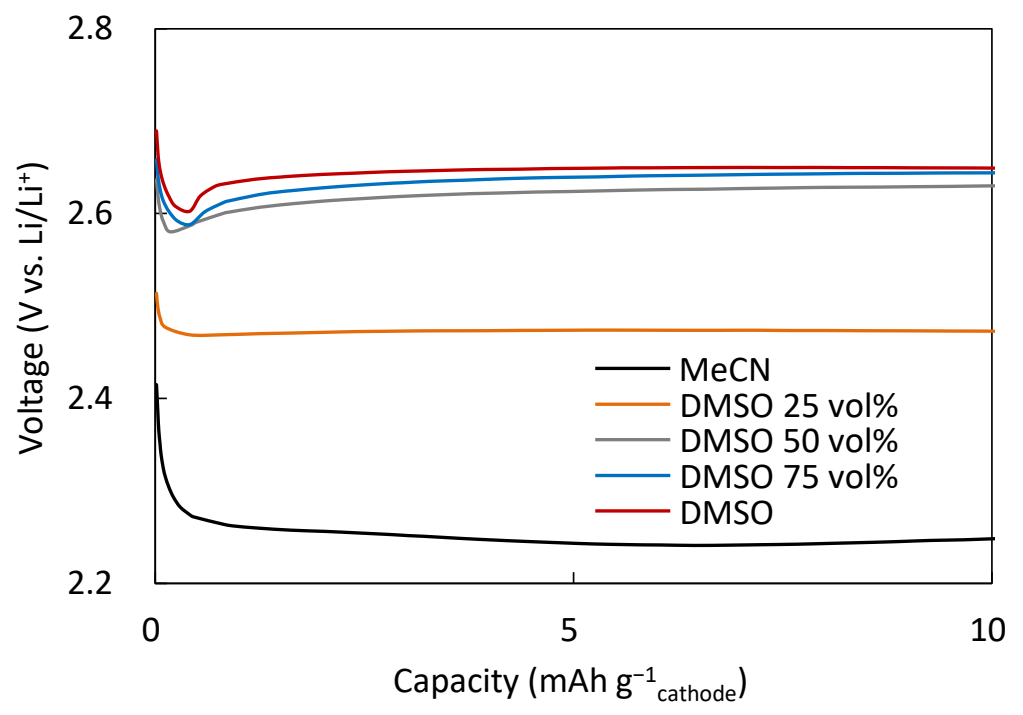

Figure S2. The expanded image of Figure 1b. The cell voltages were fluctuated at the onset of galvanostatic discharging, therefore, the LSV measurement (Figure 1c) were needed to estimate accurately the onset cell voltages of discharging. 
(a)

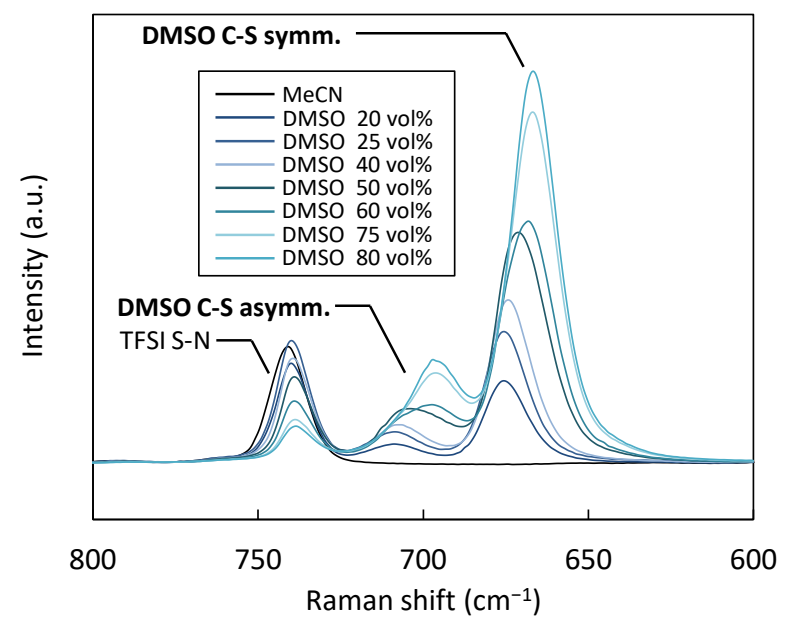

(c)

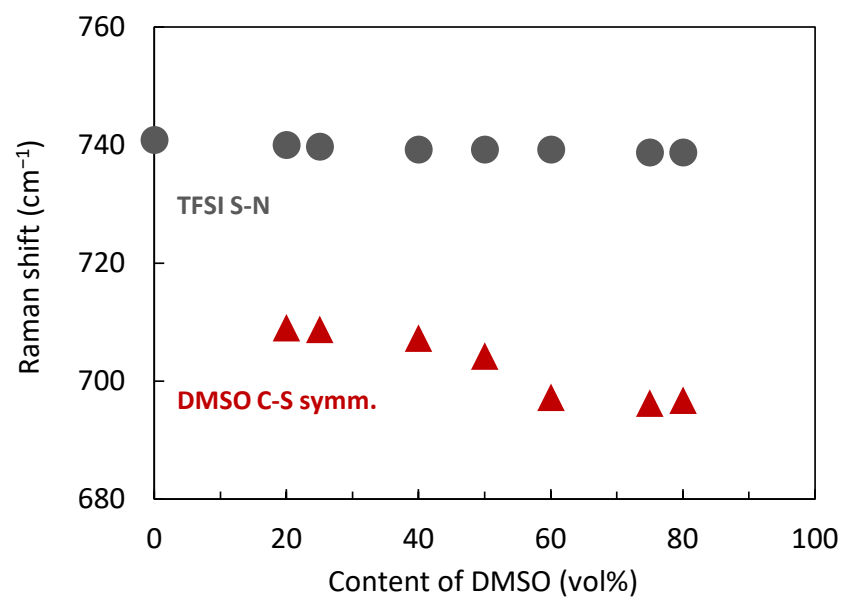

(b)

Molar ratio of DMSO/LiTFSI

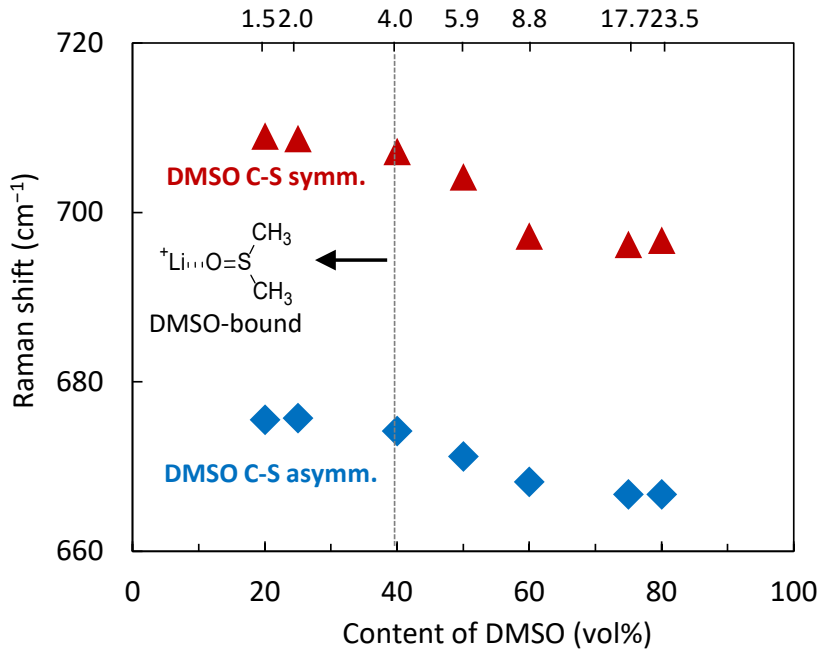

(d)

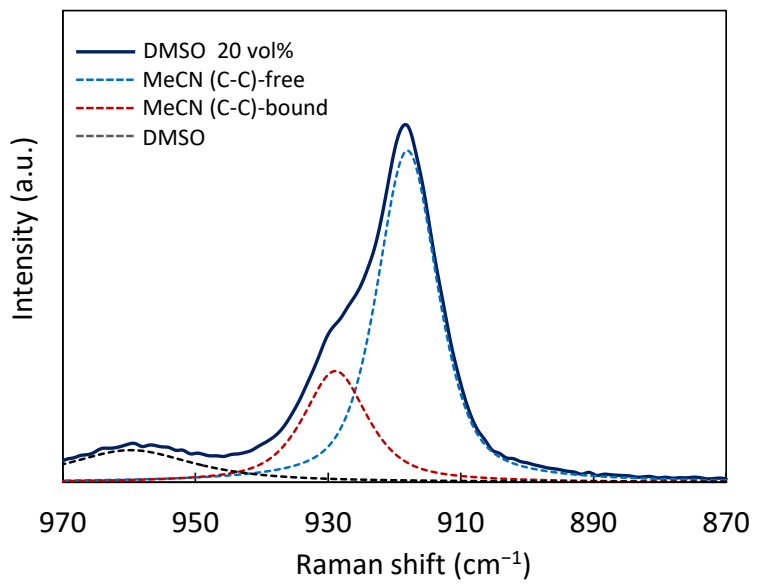

Figure S3. (a) Raman spectra of the electrolytes containing LiTFSI and the binary solvents combined with $\mathrm{MeCN}$ and DMSO in various volumetric ratio. The molar ratio of MeCN/LiTFSI was fixed on 8. (b) Peak positions of the C-S symmetric and asymmetric stretching modes of DMSO-bound and DMSO-free estimated from (a). The Raman shifts of symmetric and asymmetric C-S stretching of DMSO were gradually shifted to lower wavenumbers with increasing the content of DMSO in the electrolytes due to the increased proportion of free-DMSO. (c) The Raman shift of TFSI $^{-}$was marginally shifted to the lower wavenumbers $\left(2.1 \mathrm{~cm}^{-1}\right)$ with the increase of DMSO. These results meant that the ratio of TFSI-free increased with decrease of the concentration of TFSI $^{-}$, while the ratio of TFSI-bound species was little even in a sample with the highest concentration. $^{2}$ (d) Raman spectrum of LiTFSI and binary solvent containing $20 \mathrm{vol} \%$ of DMSO. The spectrum was deconvoluted into 3 bands. 
(a)

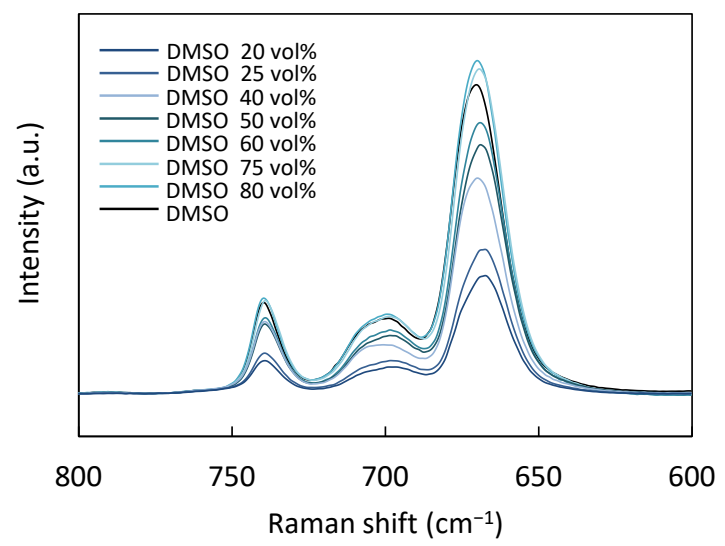

(c)

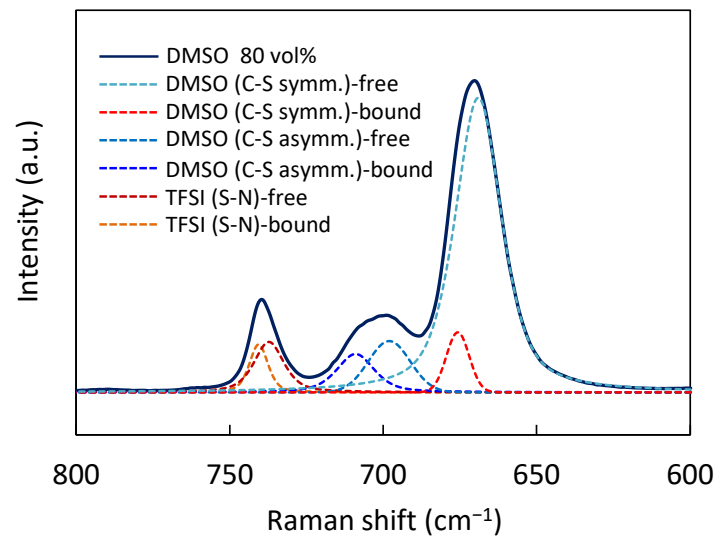

(b)

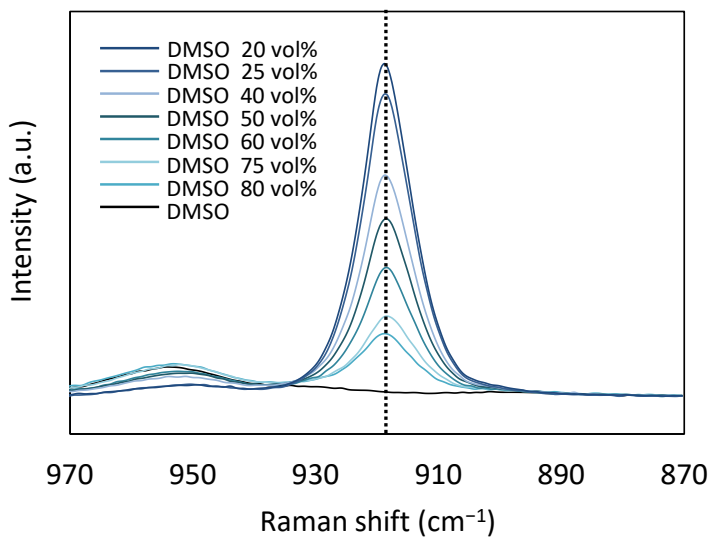

(d)

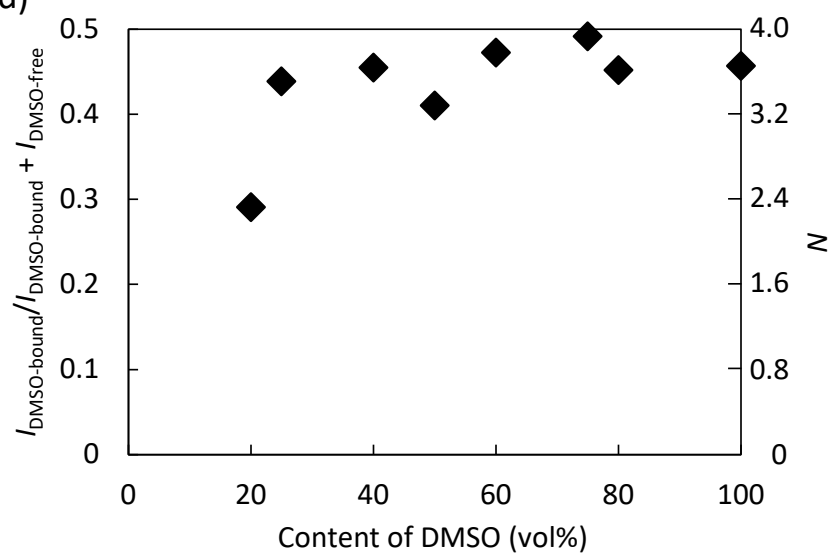

Figure S4. ( $a$ and b) Raman spectra of DMSO, and the electrolytes containing LiTFSI in the binary solvents combined with $\mathrm{MeCN}$ and DMSO in various volumetric ratio. The molar ratio of DMSO/LiTFSI was fixed on 8. (c) Raman spectrum of LiTFSI and binary solvent containing 80 vol\% of DMSO. The spectrum was deconvoluted into 6 bands. (d) Fraction of the integrated intensities of Raman peaks corresponding to DMSO-bound, $I_{\text {DMSO-bound }} /\left(I_{\text {DMSO-bound }}+I_{\text {DMSO-free }}\right)$, in the LiTFSI/binary solvent of MeCN and DMSO estimated from the Raman spectra of (b). $I_{\text {DMSO-free }}$ and $I_{\text {DMSO-bound }}$ are the integrated intensities of free and bound DMSO (C-S asymm.) peaks at 695 $\mathrm{cm}^{-1}$ and $705 \mathrm{~cm}^{-1}$, respectively. In contrast to the average solvation numbers of $N$ for MeCN shown in Figure 3c, the $N$ ranged in 3.2 to 4.0 when above 25 vol\% of DMSO was involved in the binary electrolytes. 
(a)

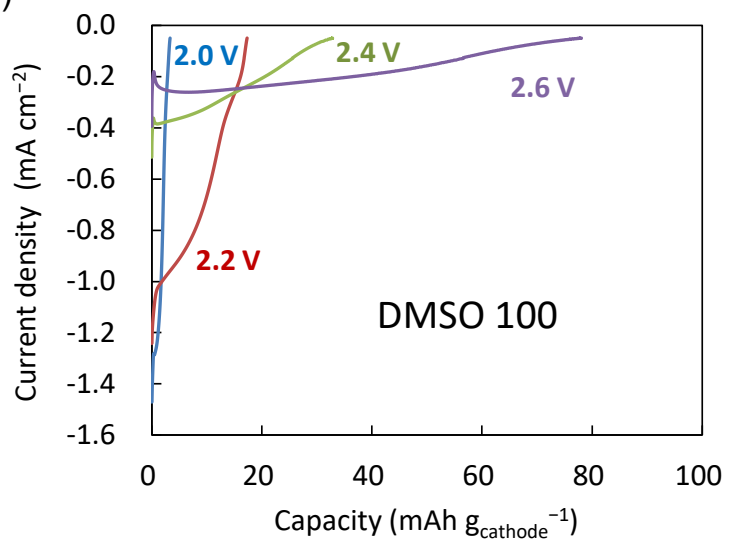

(c)

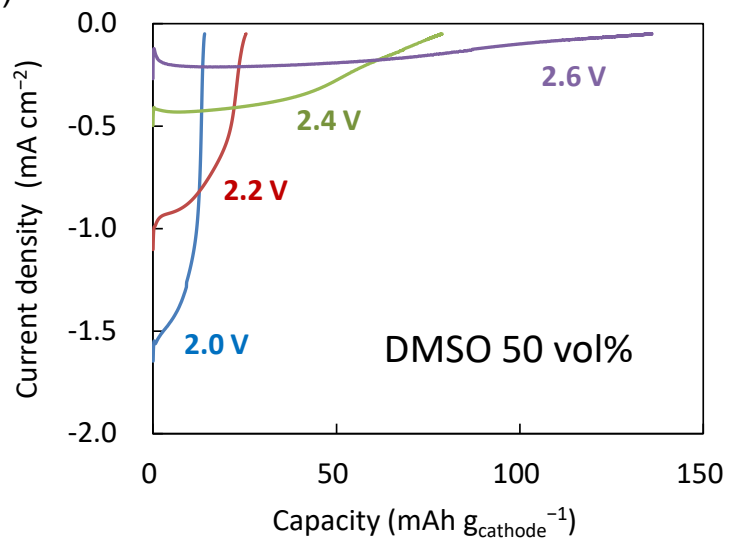

(b)

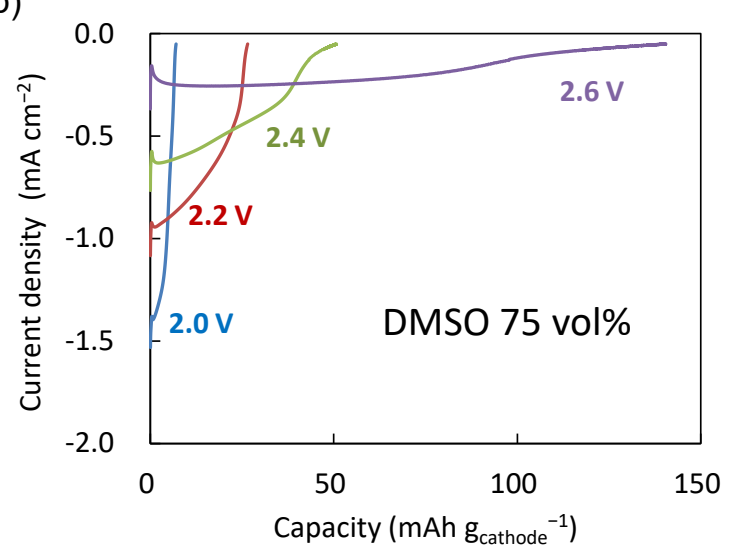

(d)

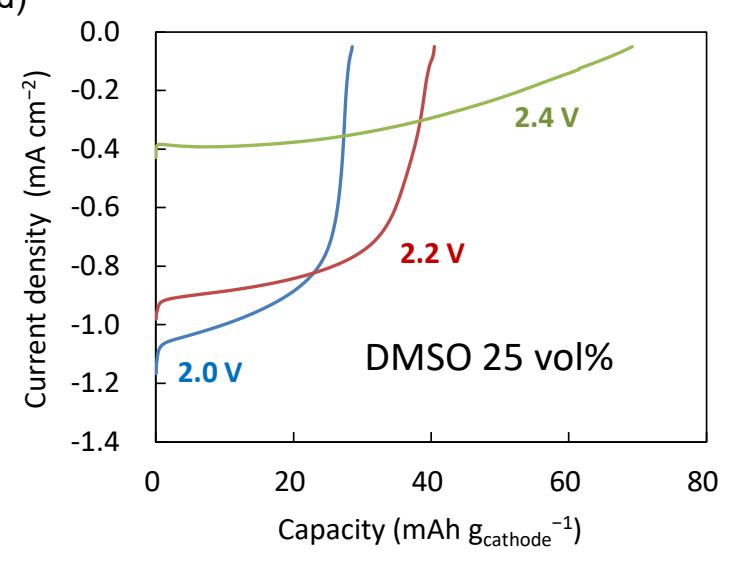

Figure S5. Potentiostatic discharging curves obtained in the binary electrolytes of $0.5 \mathrm{M}$ LiTFSI/solvent at various cell voltages. The content of DMSO in solvents are $100 \mathrm{vol} \%$ (a), 75 vol\% (b), 50 vol\% (c), and $25 \mathrm{vol} \%$ (d). 